\title{
Modified Hybrid Bronchoscope Tracking Based on Sequential Monte Carlo Sampler: Dynamic Phantom Validation
}

\author{
Xióngbiāo Luó ${ }^{1}$, Tobias Reichl² ${ }^{2}$ Marco Feuerstein ${ }^{2}$, \\ Takayuki Kitasaka ${ }^{3}$, and Kensaku Mori ${ }^{4,1}$ \\ ${ }^{1}$ Graduate School of Information Science, Nagoya University, Japan \\ ${ }^{2}$ Computer Aided Medical Procedures, Technische Universität München, Germany \\ ${ }^{3}$ Faculty of Information Science, Aichi Institute of Technology, Japan \\ ${ }^{4}$ Information and Communications Headquarters, Nagoya University, Japan
}

\begin{abstract}
This paper presents a new hybrid bronchoscope tracking method that uses an electromagnetic position sensor, a sequential Monte Carlo sampler, and its evaluation on a dynamic motion phantom. Since airway deformation resulting from patient movement, respiratory motion, and coughing can significantly affect the rigid registration between electromagnetic tracking and computed tomography (CT) coordinate systems, a standard hybrid tracking approach that initializes intensitybased image registration with absolute pose data acquired by electromagnetic tracking fails when the initial camera pose is too far from the actual pose. We propose a new solution that combines electromagnetic tracking and a sequential Monte Carlo sampler to address this problem. In our solution, sequential Monte Carlo sampling is introduced to recursively approximate the posterior probability distributions of the bronchoscope camera motion parameters in accordance with the observation model based on electromagnetic tracking. We constructed a dynamic phantom that simulates airway deformation to evaluate our proposed solution. Experimental results demonstrate that the challenging problem of airway deformation can be robustly modeled and effectively addressed with our proposed approach compared to a previous hybrid method, even when the maximum simulated airway deformation reaches $23 \mathrm{~mm}$.
\end{abstract}

\section{Introduction}

During minimally invasive diagnosis and surgery of lung and bronchus cancer, bronchoscopy is a useful tool that enables physicians to perform transbronchial biopsies (TBB) to obtain samples of suspicious tumors and to treat or remove precancerous tissue. However, it is still difficult to properly localize the biopsy needle in the region of interest (ROI) to sample tissue inside the airway tree because the TBB procedure is usually guided by conventional bronchoscopy, which only provides $2 \mathrm{D}$ information (bronchoscopic video images) and needs to be performed inside the very complex bronchial tree structure. To deal with such limitations, navigated bronchoscopy systems have been developed to help the 
bronchoscopist by fusing pre-interventional and intra-interventional information such as 3D multi-detector CT image data and real-time bronchoscopic video to provide two fundamental functions: (1) visualization of anatomical structures beyond the bronchial walls and the anatomical names of the currently displayed branches; (2) TBB guidance by showing the planned path of the bronchoscope and localizing the current bronchoscope camera inside the airway tree.

To develop such a bronchoscopic navigation system, the exact pose of the bronchoscope camera must be tracked inside the airway tree for which many techniques have been proposed. Image registration-based methods compare the similarities between real and virtual bronchoscopic images generated from preinterventional CT data $[1,2]$. However, such an optimization procedure is constrained heavily by its initialization and bifurcation or fold information to be clearly observed on real bronchoscopic images. Sensor-based electromagnetic tracking (EMT) uses a sensing coil (sensor) attached to the tip of the bronchoscope and localized by an electromagnetic tracking system, such as the commercially available superDimension navigation system [3]. However, such navigation systems suffer from the following bottlenecks: (1) sensitivity to localization problems resulting from patient movement (i.e., airway deformation). An EMT measurement usually provides the position and orientation of the bronchoscope camera relative to a fixed, world coordinate system and hence the current measurement under airway deformation does not correspond exactly to the current bronchoscope camera pose; (2) measurement inaccuracies because of magnetic field distortion caused by ferrous metals or conductive material within or close to the working volume. To address airway deformation, Gergel et al. applied particle filtering to all camera positions and orientations acquired by EMT and projected them to a previously segmented centerline of the bronchial tree [4], so they assume a bronchoscope camera that is always moving along the centerline of the airways; however this is a hard constraint since it is easily violated by a bronchoscopist in the operating room. Otherwise, the measurement inaccuracies of EMT are difficult to correct, unless combined with optical tracking [5,6]. Furthermore, a combination of image- and sensor-based methods for bronchoscope tracking was originally proposed by Mori et al. [7]. Their hybrid method was improved by Soper et al. [8] who integrated electromagnetic tracking, image-based tracking, Kalman filtering, and a respiratory motion compensation method using a surrogate sensor. According to their evaluation of the state-of-the-art methods, the hybrid method is a promising means for bronchoscope tracking and definitely outperforms other methods.

In our paper, we modify hybrid bronchoscope tracking using a sequential Monte Carlo (SMC) sampler to improve tracking performance and to deal with the disadvantages of EMT and the restrictions of image-based methods. Bronchoscope tracking based on Bayesian or motion filtering has already been proposed in $[9,10]$. However, $[9,10]$ only focused on how to improve the initialization of image registration methods without estimating the rotational part of the bronchoscope camera motion. Our proposed method incorporates electromagnetic tracking and a sequential Monte Carlo sampler to directly estimate the posterior 
probability distribution of the current bronchoscope camera motion parameters. This modified method significantly increases the accuracy and the robustness of bronchoscope tracking, as shown in our experimental results.

\section{SMC Sampler-Based Bronchoscope Tracking}

Our modified hybrid bronchoscope tracking method consists of three stages: (1) during camera and hand-eye calibration, we apply camera calibration to obtain the intrinsic parameters of the bronchoscope camera and employ hand-eye calibration to perform electromagnetic sensor and camera alignment; (2) the CT-tophysical space registration step obtains the initial rigid registration between the EMT and CT coordinate systems. We can use a landmark-based or a landmarkfree method to calculate this transformation; (3) the sequential Monte Carlo sampler-based camera motion estimation stage estimates the posterior probability distribution of the current bronchoscope camera motion parameters and determines the estimated camera pose at the maximal probability to correspond to the current bronchoscope camera pose.

Since the first two stages of the proposed method closely resemble the work of Luo et al. [11], we do not describe them here. We focus on modeling and predicting the bronchoscope camera motion based on a sequential Monte Carlo sampler and electromagnetic tracking.

Sequential Monte Carlo samplers such as frameworks [12-14] are a generalized class of algorithms dealing with the state estimation problem for nonlinear/nonGaussian dynamic systems that sequentially sample a set of weighted particles from a sequence of probability distributions defined upon essentially arbitrary spaces using importance sampling and resampling mechanisms. They have been used previously for vision on the basis of structure from motion (SFM), for example, the usage of a general Monte Carlo sampler for SFM in the work of Forsyth et al. [15] and the investigation of particle filtering for simultaneous localization and mapping (SLAM) in [16].

Generally, sequential Monte Carlo samplers are quite similar: samples are determinately drifted and stochastically diffused to approximate the posterior probability distributions of interest. We use an SMC sampler, which resembles the approach of Qian et al. in [17], and only sample the 3-D camera motion parameters; however, Qian et al. sampled the feature correspondences for motion depth determination. We use sequential importance sampling with resampling (SIR) at each iteration to estimate the posterior probability distribution of current bronchoscope camera motion.

\subsection{SMC Sampler}

Before camera motion estimation, in this section, we briefly review the sequential Monte Carlo sampler based on the SIR scheme.

Suppose a set of state vectors $\mathcal{X}_{i}=\left\{\mathbf{x}_{i}: i=1, \ldots, N\right\}$ and similarly a set of measurements with their history $\mathcal{Y}_{i}=\left\{\mathbf{y}_{i}: i=1, \ldots, N\right\}$, where $N$ is the number 
of states or measurements. The sampler using the SIR scheme constructs and approximates the posterior probability distribution $p\left(\mathbf{x}_{i} \mid \mathcal{Y}_{i}\right)$ of the current state vector $\mathbf{x}_{i}$, given all available information, for example, the previous posterior probability distribution $p\left(\mathbf{x}_{i-1} \mid \mathcal{Y}_{i-1}\right)$. To estimate $p\left(\mathbf{x}_{i} \mid \mathcal{Y}_{i}\right)$, the SIR algorithm first generates a set of random samples $\mathcal{X}_{i}^{k}=\left\{\mathbf{x}_{i}^{k}: k=1, \ldots, M\right\}$ with associated weights $\mathcal{W}_{i}^{k}=\left\{w_{i}^{k}: k=1, \ldots, M\right\}$ ( $M$ is the sample size) at time $i$ based on the previous posterior probability distribution $p\left(\mathbf{x}_{i-1} \mid \mathcal{Y}_{i-1}\right)$ and the current measurement $\mathbf{y}_{i}$. After that, $p\left(\mathbf{x}_{i} \mid \mathcal{Y}_{i}\right)$ is approximated by these samples with respect to $\mathbf{x}_{i}^{k}$ and $w_{i}^{k}[13]$ :

$$
p\left(\mathbf{x}_{i} \mid \mathcal{Y}_{i}\right) \approx \sum_{k=1}^{M} w_{i}^{k} \delta\left(\mathbf{x}_{i}-\mathbf{x}_{i}^{k}\right),
$$

where $\delta(\cdot)$ is the Dirac delta function. $w_{i}^{k}$ can be calculated by

$$
w_{i}^{k} \propto w_{i-1}^{k} \frac{p\left(\mathbf{y}_{i} \mid \mathbf{x}_{i}^{k}\right) p\left(\mathbf{x}_{i}^{k} \mid \mathbf{x}_{i-1}^{k}\right)}{q\left(\mathbf{x}_{i}^{k} \mid \mathbf{x}_{i-1}^{k}, \mathbf{y}_{i}\right)},
$$

where the proposal $q(\cdot)$ is called an importance density function that affects the degree of sample degeneracy. Usually, it is convenient to choose $q(\cdot)$ as the prior: $q\left(\mathbf{x}_{i}^{k} \mid \mathbf{x}_{i-1}^{k}, \mathbf{y}_{i}\right)=p\left(\mathbf{x}_{i}^{k} \mid \mathbf{x}_{i-1}^{k}\right)$, then $w_{i}^{k} \propto w_{i-1}^{k} p\left(\mathbf{y}_{i} \mid \mathbf{x}_{i}^{k}\right)$ [13].

Basically, a pseudo-code description of an SMC sampler using SIR can be generalized in Algorithm 1 as follows:

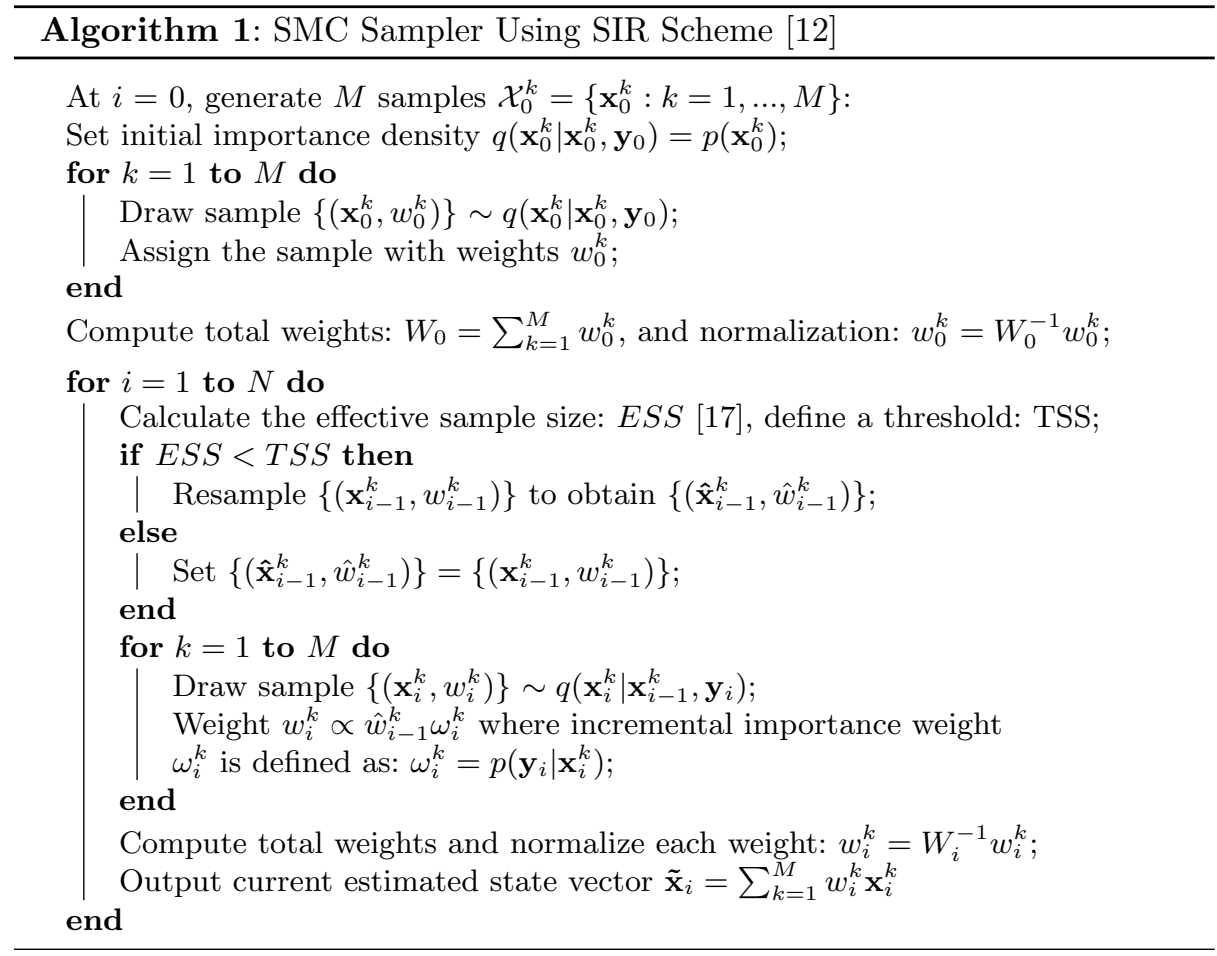




\subsection{Definitions of Bronchoscopic Camera Motion}

We must define the coordinate systems to be used since bronchoscope tracking seeks a transformation matrix ${ }^{C T} \mathbf{T}_{C}$ including translation ${ }^{C T} \mathbf{t}_{C}$ and rotation ${ }^{C T} \mathbf{R}_{C}$ from the bronchoscope camera coordinate system to the CT coordinate system. Fig. 1 outlines the relationships and transformation matrices between each coordinate system. ${ }^{F} \mathbf{T}_{S}$ describes the relationship between the sensor and magnetic field coordinate systems. ${ }^{W} \mathbf{T}_{F}$ is from the magnetic field coordinate system to the world coordinate system, and ${ }^{C T} \mathbf{T}_{W}$ is from the world coordinate system to the CT coordinate system. We formulate the relationship between the sensor and world coordinate systems as ${ }^{W} \mathbf{T}_{S}^{(i)}={ }^{W} \mathbf{T}_{F}{ }^{F} \mathbf{T}_{S}^{(i)}$, where ${ }^{F} \mathbf{T}_{S}^{(i)}$ is the $i$-th sensor output. Additionally, the transformation between the camera and the sensor (both attached at the bronchoscope tip) is represented by ${ }^{S} \mathbf{T}_{C}$.

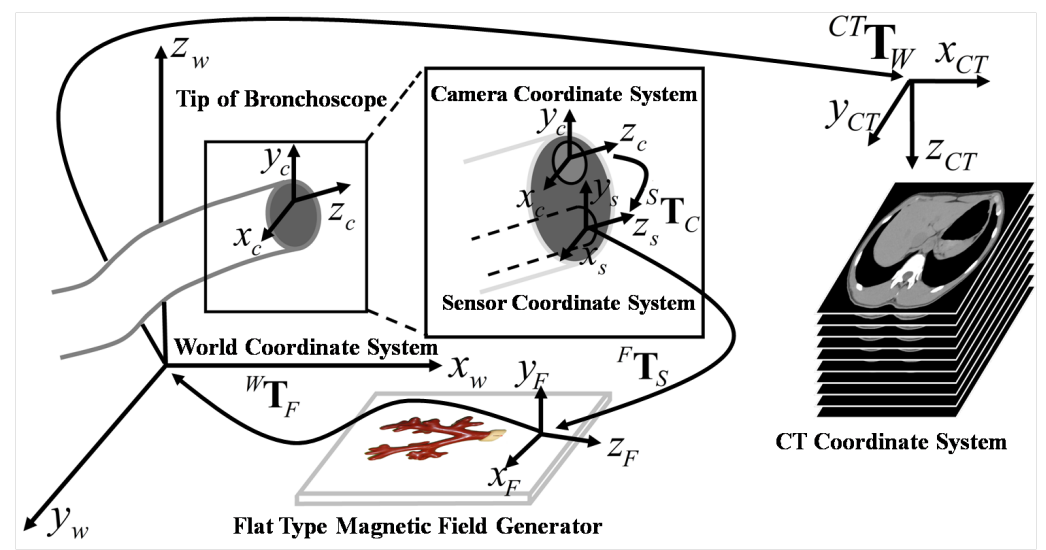

Fig. 1: Relationship between coordinate systems in our navigated bronchoscopy.

In our study, we use the SMC sampler to predict the posterior probability distributions for the bronchoscope camera pose parameters. The camera motion state is described by translation ${ }^{C T} \mathbf{t}_{C}$ and rotation ${ }^{C T} \mathbf{R}_{C}$ from the bronchoscope camera coordinate system to the CT coordinate system. For the rotation part, we use a quaternion but not a rotation matrix ${ }^{C T} \mathbf{R}_{C}$ in our implementation. The quaternion has been demonstrated to be very powerful to characterize the rotation part since it has such advantages as compactness and the avoidance of discontinuous jumps compared to other representations (e.g., Euler angles).

A quaternion representation of rotation can be conveniently considered as a normalized vector with four components:

$$
\mathbf{q}=\left[\begin{array}{llll}
q_{0} & q_{x} & q_{y} & q_{z}
\end{array}\right], \quad q_{0}{ }^{2}+q_{x}{ }^{2}+q_{y}{ }^{2}+q_{z}{ }^{2}=1 .
$$

Global motion state $\mathbf{x}_{i}$ that corresponds to the current camera frame can be parameterized by a seven-dimensional vector:

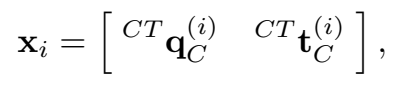


where $i$ means the camera motion state at time $i$ or denotes the $i$-th electromagnetic tracking result.

According to a sequential Monte Carlo sampler, each random sample $\left(\mathbf{x}_{i}^{k}, w_{i}^{k}\right)$ represents a potential pose of the bronchoscope camera and involves an important weight defined as the similarities between the real and virtual bronchoscopic images in our case. A random sample set $\mathcal{S}_{i}^{k}=\left\{\left(\mathbf{x}_{i}^{k}, w_{i}^{k}\right): k=1,2,3, \ldots, M\right\}$ is used to approximate the posterior probabilistic density of the current bronchocope camera pose at time $i$.

\subsection{SMC Sampler for Camera Motion Estimation}

Our proposed hybrid bronchoscope camera motion tracking process is mainly performed by the following steps described in this section.

After parameterizing the current camera motion state $\mathbf{x}_{i}$ involved with the SMC sampler, bronchoscope tracking continuously estimates the posterior probability distribution $p\left(\mathbf{x}_{i} \mid \mathcal{Y}_{i}\right)$ using a set of random samples $\mathcal{S}_{i}^{k}$, where the sample weights are proportional to $p\left(\mathbf{y}_{i} \mid \mathbf{x}_{i}^{k}\right)$, as defined in Algorithm 1. To obtain these random samples $\mathcal{S}_{i}^{k}$, the $\mathrm{SMC}$ sampler requires the probabilistic model $p\left(\mathbf{x}_{i}^{k} \mid \mathbf{x}_{i-1}^{k}\right)$ for the state dynamic between the time steps and likelihood function (or an important density function) $q\left(\mathbf{x}_{i}^{k} \mid \mathbf{x}_{i-1}^{k}, \mathbf{y}_{i}\right)$ for the observations (or measurements) shown in Eq. 2. Additionally, to characterize a random sample $\mathcal{S}_{i}^{k}$, the weight $w_{i}^{k}$ also needs to be determined by incremental importance weight $\omega_{i}^{k}$ that equals $p\left(\mathbf{y}_{i} \mid \mathbf{x}_{i}^{k}\right)$. Therefore, the following steps are implemented for the SMC sampler to estimate the bronchoscope camera motion.

[Step 1] State Dynamic. During this state transition step, the bronchoscope motion dynamic at frame $i$ is usually characterized as a second order process that is described by a second order difference equation [17]

$$
\mathbf{x}_{i}^{k}=U \mathbf{x}_{i-1}^{k}+V \mathbf{n}_{i}^{k}
$$

where the matrix $U$ describes the deterministic drift part of the state dynamic model and depends on the EMT measurements $\mathbf{y}_{i}$ and $\mathbf{y}_{i-1}$ while the matrix $V$ represents the stochastic diffusion component of the state dynamic model or describes the uncertainty of inter-frame camera motion defined on the basis of Eq. 4. We note that $\mathbf{n}_{i}^{k}$ is an independent stochastic variable or a noise term that is discussed in the following paragraph.

Since we have no prior knowledge of the bronchoscope camera movement, we utilize a random walk model to characterize $p\left(\mathbf{x}_{i}^{k} \mid \mathbf{x}_{i-1}^{k}\right)$ for the pointwise state evaluation. As bronchoscopic frames are used as image sources, the changes of the motion parameters are usually quite small. For example, in our case the frame rate of the bronchoscope camera is 30 frames per second; however, the typical moving speed of the camera is around $10 \mathrm{~mm}$ per second, so the magnitude of inter-frame motion changes at $0.33 \mathrm{~mm}$ per second. Therefore, we used a random walk on the basis of normal density with respect to noise vector $\mathbf{n}_{i}^{k}$ : $\mathbf{n}_{i}^{k} \sim \mathcal{N}\left(\mu, \sigma^{2}\right)$ to approximate the state dynamic in accordance with Eq. 5 [18]: 


$$
p\left(\mathbf{x}_{i}^{k} \mid \mathbf{x}_{i-1}^{k}\right) \propto \frac{1}{\sqrt{2 \pi \sigma}} \exp \left(-\left(V^{-1}\left(\mathbf{x}_{i}^{k}-U \mathbf{x}_{i-1}^{k}\right)-\mu\right)^{2} / 2 \sigma^{2}\right),
$$

After undergoing a random walk based on normal density, the drifted and diffused state $\mathbf{x}_{i}^{k}$ has a probabilistic distribution in accordance with Eq. 6 .

[Step 2] Observation Model. A good choice of the important density function $q\left(\mathbf{x}_{i}^{k} \mid \mathbf{x}_{i-1}^{k}, \mathbf{y}_{i}\right)$ can alleviate the sample degeneracy problem. In the SIR algorithm, it is appropriately chosen as prior density $p\left(\mathbf{x}_{i}^{k} \mid \mathbf{x}_{i-1}^{k}\right)$ [13], as mentioned above. We follow this choice: $q\left(\mathbf{x}_{i}^{k} \mid \mathbf{x}_{i-1}^{k}, \mathbf{y}_{i}\right)=p\left(\mathbf{x}_{i}^{k} \mid \mathbf{x}_{i-1}^{k}\right)$. Therefore, in our case, the observation density $p\left(\mathbf{y}_{i} \mid \mathbf{x}_{i}\right)$ can be decided by:

$$
p\left(\mathbf{y}_{i} \mid \mathbf{x}_{i}=\mathbf{x}_{i}^{k}\right) \propto w_{i}^{k}\left(\sum_{j=1}^{M} w_{i}^{j}\right)^{-1} .
$$

We clarify that the observation $\mathbf{y}_{i}$ is defined as the EMT measurement and modeled as $\mathbf{y}_{i}=H \mathbf{x}_{i}$, where $H$ is the observation matrix and is usually defined as the transformation from the CT to the EMT coordinates.

[Step 3] Determination of Sample Weight. During the two steps described above, a sample weight $w_{i}^{k}$ must be computed to assess the sample performance.

In our study, a sample weight $w_{i}^{k}$ is defined as the similarity between the current real bronchoscopic image $\mathbf{I}_{R}^{(i)}$ and the virtual bronchoscopic image $\mathbf{I}_{V}$ generated using estimated virtual camera parameters $\mathbf{x}_{i}^{k}$ based on a volume rendering technique. Based on the selective image similarity measure [2], after the division of images $\mathbf{I}_{R}^{(i)}$ and $\mathbf{I}_{V}$ into subblocks and the selection of subblocks, we use a modified mean squared error (MoMSE) to calculate the similarity:

$$
\operatorname{MoMSE}\left(\mathbf{I}_{R}^{(i)}, \mathbf{I}_{V}\right)=\frac{1}{\left|A^{(i)}\right|} \sum_{D \in A^{(i)}} \frac{1}{|D|} \sum_{D}\left(\left(\mathbf{I}_{R}^{(i)}-\overline{D \mathbf{I}_{R}^{(i)}}\right)-\left(\mathbf{I}_{V}-\bar{D} \mathbf{I}_{V}\right)\right)^{2}
$$

where $\left|A^{(i)}\right|$ is the number of selected subblocks in the list of selected subblocks $A^{(i)}$, and $\overline{D \mathbf{I}_{R}^{(i)}}$ and $\overline{\mathbf{V}_{D}}$ are the respective mean intensities of all subblocks $D$ of $\mathbf{I}_{R}^{(i)}$ and $\mathbf{I}_{V}$. The mean intensities of $\mathbf{I}_{R}^{(i)}$ and $\mathbf{I}_{V}$ may be different in an actual bronchoscopic image because of the different strengths of the light sources. To reduce this effect, $\overline{D \mathbf{I}_{R}^{(i)}}$ and $\overline{D \mathbf{I}_{V}}$ are subtracted from each pixel.

The weight $w_{i}^{k}$ can be formulated as

$$
w_{i}^{k}=\operatorname{MoMSE}\left(\mathbf{I}_{R}^{(i)}, \mathbf{I}_{V}\left(\mathbf{x}_{i}^{k}\right)\right)
$$

Finally, in our case, the output of the SMC sampler for the current estimated motion state can be determined in accordance with $w_{i}^{k}$ :

$$
\tilde{\mathbf{x}}_{i}=\arg \max _{w_{i}^{k}}\left\{\left(\mathbf{x}_{i}^{k}, w_{i}^{k}\right)\right\}
$$


that is, sample $\tilde{\mathbf{x}}_{i}$ with maximal weight $\tilde{w}_{i}$ corresponds to the maximal similarity between the current bronchoscope camera frame and the generated virtual frame.

Our modified hybrid bronchoscope tracking based on an SMC sampler can be summarized in Algorithm 2 as follows.

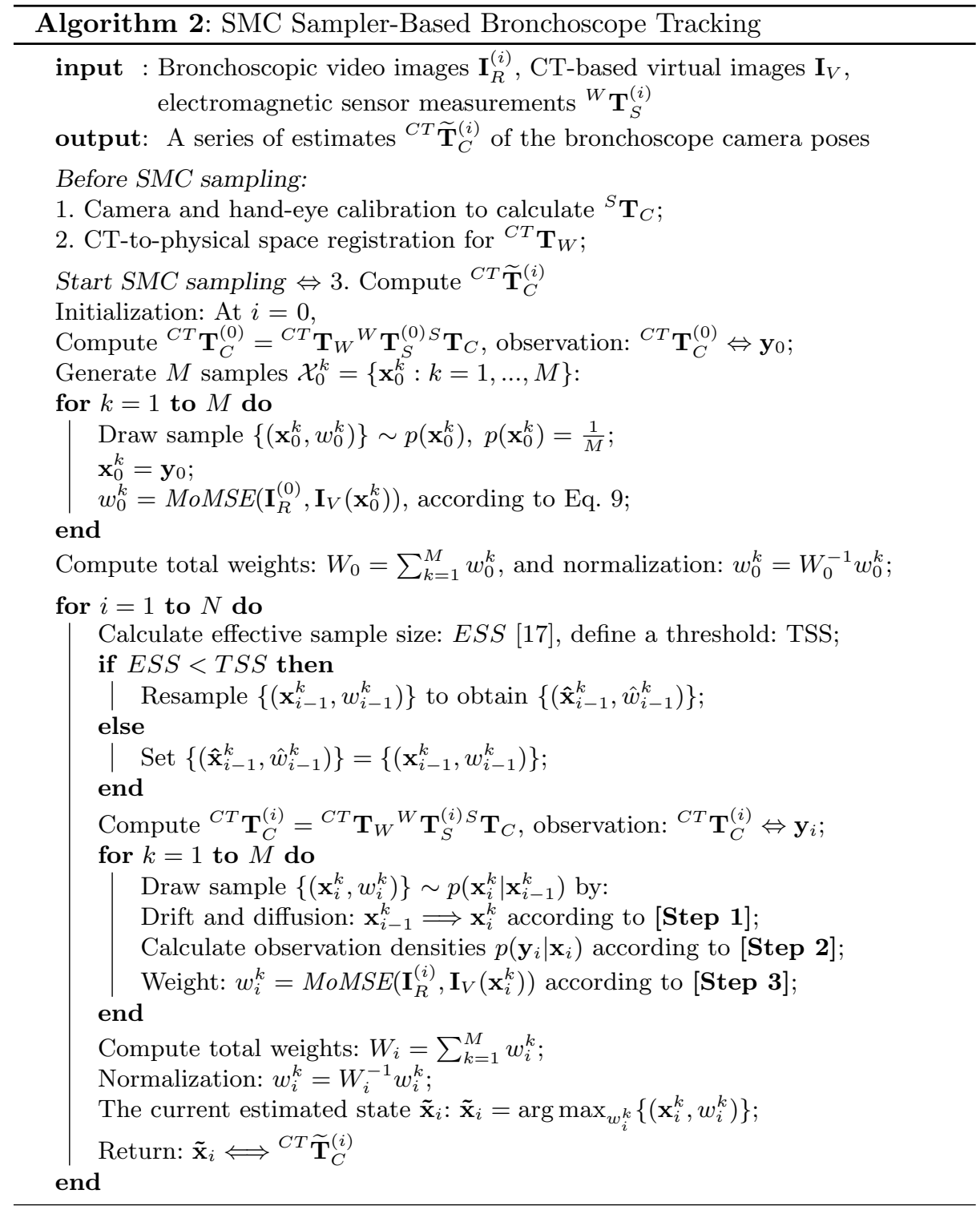

\section{Experimental Results}

For evaluating the performance of our proposed tracking method, we manufactured a dynamic bronchial phantom (Fig. 2) to simulate breathing motion. 
We connected the rubber phantom to a motor using nylon threads. A LEGO Mindstorm (LEGO, Denmark) was utilized as power source to generate movement. With the controller part (NXT: a programmable robotics kit included in LEGO Mindstorm), we can manipulate the motor motion including the directions and the rotational speeds. The phantom simulates respiratory motion when the thread changes its length. We can adjust the amount of simulated motion, and its maximum deformation is about $24 \mathrm{~mm}$.

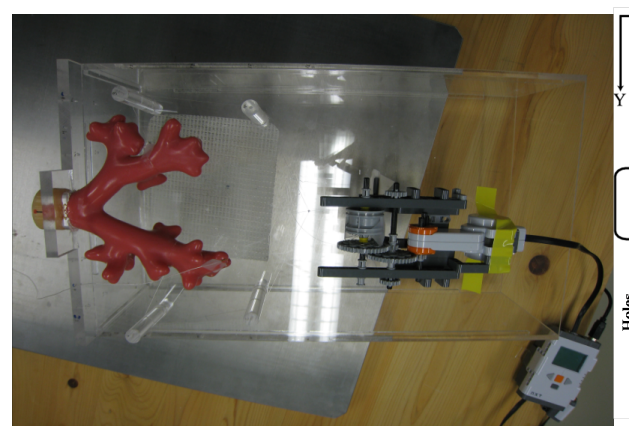

(a)

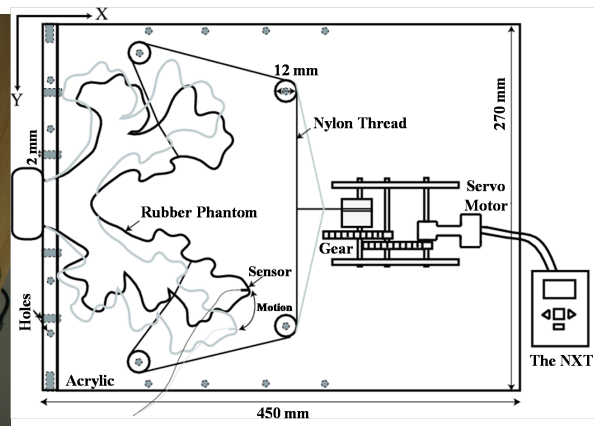

(b)

Fig. 2: Dynamic motion phantom: (a) picture of real phantom and (b) drawing of phantom movement.

For dynamic phantom validation, we compare four tracking schemes: (a) Solomon et al. [3], only using EMT, (b) Mori et al. [7], intensity-based image registration directly initialized by the EMT results, (c) Luo et al [11], the better one of two proposed schemes in [11], and (d) our method, as described in Section 2.3.

Table 1: Comparison of registered results (the unit of maximal motion is $\mathrm{mm}$ )

\begin{tabular}{|c|c|c|c|c|c|}
\hline \multirow{2}{*}{$\begin{array}{l}\text { Experi. } \\
\text { (frames) }\end{array}$} & \multirow{2}{*}{$\begin{array}{l}\text { Maximal } \\
\text { motion }\end{array}$} & \multicolumn{4}{|c|}{ Number (percentage) of successfully registered frames } \\
\hline & & Solomon et al. [3] & Mori et al. [7] & Luo et al. [11] & Our method \\
\hline $\mathrm{A}(1285)$ & 6.13 & $850(66.1 \%)$ & $958(74.6 \%)$ & $1034(80.5 \%)$ & $1224(95.3 \%)$ \\
\hline $\mathrm{B}(1326)$ & 11.82 & $783(59.0 \%)$ & $863(65.1 \%)$ & $1018(76.8 \%)$ & $1244(93.8 \%)$ \\
\hline $\mathrm{C}(1573)$ & 18.75 & $894(56.8 \%)$ & $972(61.8 \%)$ & $1153(73.3 \%)$ & $1431(91.0 \%)$ \\
\hline $\mathrm{D}(1468)$ & 23.61 & $716(48.8 \%)$ & $850(57.9 \%)$ & $1036(70.6 \%)$ & $1300(88.6 \%)$ \\
\hline & $\operatorname{tal}(5652)$ & $3243(57.4 \%)$ & $3643(64.5 \%$ & $4241(75.0 \%)$ & $5199(92.0 \%)$ \\
\hline
\end{tabular}

Table 1 shows the quantitative results of the evaluation of the methods. Here we counted the number of frames that were successfully registered by visually inspecting the similarities between the real and virtual images. The maximum simulated respiratory motion for different experiments is also shown in Table 1. Our proposed method significantly improved the tracking performance. Furthermore, examples of experiments C and D for successfully registered frames 
are displayed in Fig. 3, which shows examples of real bronchoscopic (RB) images and corresponding virtual bronchoscopic (VB) images generated from the camera parameters predicted by each method.

\section{Discussion}

The objective of this study is to design and improve the performance of hybrid bronchoscope tracking under airway deformation during bronchoscopic navigation, in particular, to deal with the limitations of electromagnetic tracking. We used a sequential Monte Carlo sampler to modify previous hybrid bronchoscope tracking methods. According to the experimental results, the posterior probability distributions of the bronchocope camera poses are almost completely approximated using the sequential Monte Carlo sampler. Hence we improved our previous proposed hybrid tracking methods $[7,11]$ in various aspects.

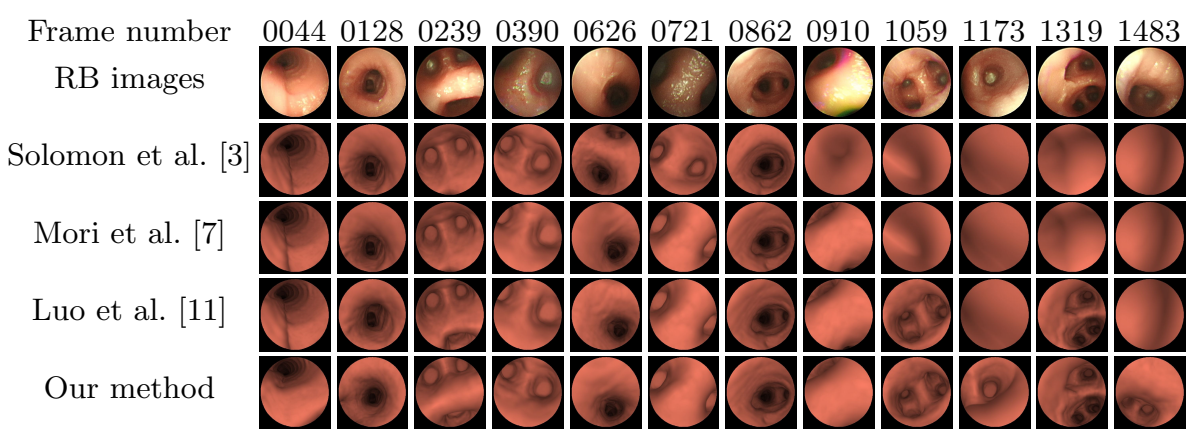

(a) Examples of experiment $\mathrm{C}$

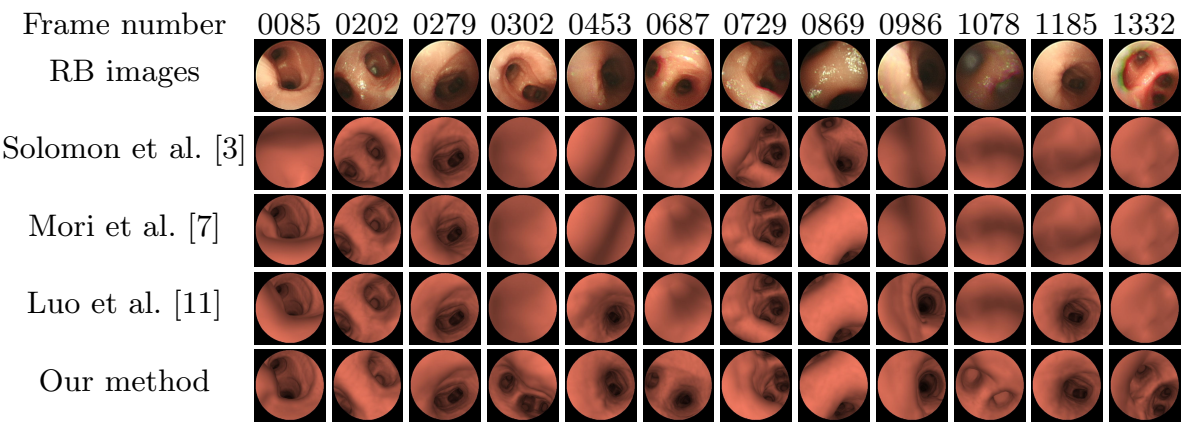

(b) Examples of experiment D

Fig. 3: Results of bronchoscope tracking for different methods under simulated breathing motion using our dynamic phantom. The top row shows selected frame numbers and the second row shows their corresponding phantom RB images. The other rows display virtual bronchoscopic images generated from tracking results using the methods of Solomon et al. [3], Mori et al. [7], Luo et al. [11], and our method. Our proposed method shows the best performance. 
As for the previous hybrid method [7], its tracking robustness and accuracy usually suffer from the following: (1) dependencies on the initialization of image registration and visible characteristic structures (i.e., folds or bifurcations of the bronchi) for similarity computation; (2) airway deformation, in particular respiratory motion. For the registration step (an optimization procedure), the optimizer is unavoidably trapped in local minima. We have already addressed these limitations and improved the tracking performance by modifying the initialization of image registration in our previous work [11]. In this study, our modified method was more effectively disengaged from these constraints using a sequential Monte Carlo sampler, compared to our previous methods $[7,11]$. We greatly approximate the posterior densities of the state parameters by collecting a set of random samples and sequentially predict the camera motion parameters on the basis of the importance sampling, which provides the ability to maintain potential importance modes that either they are confirmed or moved to be the subsequent observations. This results in our proposed method that can avoid the optimization registration algorithm which is trapped in local minima in most cases and particularly has the ability to automatically retrieve the tracking loss even in case of image artifacts. Hence, our method shows the best tracking performance in Table 1 and Fig. 3, compared to the previous methods.

However, in our experiments, the modified methods still failed to correctly register all $\mathrm{RB}$ and $\mathrm{VB}$ frames when continuously tracking the bronchoscope for the following reasons: (1) the dynamic error of EMT (because of the ferrous material contained inside the bronchoscope), as mentioned in Section 1, affected the observation accuracy; (2) our simulated breathing motion is rather big and not realistic enough. Currently it is only in the left-right and superior-inferior directions for the peripheral lung. The trachea does not move. The magnitude of the motion can be adjusted to $6 \sim 24 \mathrm{~mm}$. However, for a real patient, respiratory motion is greatest in the superior-inferior direction $(\sim 9 \mathrm{~mm})$, moderate in the anterior-posterior direction $(\sim 5 \mathrm{~mm})$, and lowest in the left-right direction $(\sim 1$ $\mathrm{mm})$ [19].

Additionally, the average runtime of our proposed method per frame (1.7 seconds) is higher than that of the previous hybrid method ( 0.5 seconds), because each random sample must compute its weight based on the similarities between real and virtual images; this is really time-consuming.

\section{Conclusions and Future Work}

This paper presented a modified hybrid bronchoscope tracking method that used an electromagnetic position sensor and a sequential Monte Carlo sampler and evaluation on a dynamic phantom. We used a sequential Monte Carlo sampler to approximate the posterior probability distributions of the bronchoscope camera motion parameters. Experimental results demonstrated that the modified method gives impressive approximations to the bronchoscope camera motion and successfully registered a total of 5199 (92.0\%) bronchoscopic images, increasing the tracking performance by $17.0 \%$ compared to the state-of-the-art hybrid method. We conclude that our method significantly alleviates the sensitivity to 
the localization problems of electromagnetic tracking that usually result from airway deformation, particularly respiratory motion. Our future work includes experiments on patient datasets using our proposed method in the operating room and improvement of its computational efficiency.

\section{References}

1. Bricault, I., et al: Registration of real and CT-derived virtual bronchoscopic images to assist transbronchial biopsy. IEEE TMI 17 (1998) 703-714

2. Deguchi, D., et al: Selective image similarity measure for bronchoscope tracking based on image registration. MedIA 13 (2009) 621-633

3. Solomon, S.B., et al: Three-dimensionsal CT-guided bronchoscopy with a real-time electromagnetic position sensor: a comparison of two image registration methods. Chest 118 (2000) 1783-1787

4. Gergel, I., et al: Particle filtering for respiratory motion compensation during navigated bronchoscopy. In: Proceedings of SPIE. Volume 7625. (2010) 76250W

5. Deguchi, D., et al: A method for bronchoscope tracking by combining a position sensor and image registration. In: Proceedings of CARS. Volume 1281. (2005) 630-635

6. Feuerstein, M., et al: Magneto-optical tracking of flexible laparoscopic ultrasound: Model-based online detection and correction of magnetic tracking errors. IEEE TMI 28 (2009) 951-967

7. Mori, K., et al: Hybrid bronchoscope tracking using a magnetic tracking sensor and image registration. In: Proceedings of MICCAI. Volume LNCS 3750. (2005) pp.543-550

8. Soper, T.D., et al: In vivo validation of a hybrid tracking system for navigation of an ultrathin bronchoscope within peripheral airways. IEEE TBME 57 (2010) $736-745$

9. Nagao, J., et al: Fast and accurate bronchoscope tracking using image registration and motion prediction. In: Proceedings of MICCAI. Volume 3217. (2004) 551-558

10. Deligianni, F., et al: Predictive camera tracking for bronchoscope simulation with CONDensation. In: Proceedings of MICCAI. Volume 3749. (2005) 910-916

11. Luo, X., et al: Towards hybrid bronchoscope tracking under respiratory motion: evaluation on a dynamic motion phantom. In: Proceedings of SPIE. Volume 7625. (2010) $76251 \mathrm{~B}$

12. Liu, J.S., et al: Sequential Monte Carlo methods for dynamic systems. Journal of the American Statistical Association 93 (1998) 1032-1044

13. Arulampalam, M., et al: A tutorial on particle filters for nonlinear/non-gaussian Bayesian tracking. IEEE TSP 50 (2002) 174-188

14. Moral, P.D., et al: Sequential Monte Carlo samplers. Journal of the Royal Statistical Society: Series B (Statistical Methodology) 68 (2006) 411-436

15. Forsyth, D., et al: The joy of sampling. IJCV 41 (2001) 109-134

16. Pupilli, M.: Particle filtering for real-time camera localisation. PhD thesis, University of Bristol, UK (2006)

17. Qian, G., et al: Structure from motion using sequential Monte Carlo methods. IJCV 59 (2004) 5-31

18. Doucet, A., et al: On sequential monte carlo sampling methods for Bayesian filtering. Statistics and Computing 10 (2000) 197-208

19. Soper, T.D., et al: A model of respiratory airway motion for real-time tracking of an ultrathin bronchoscope. In: Proceedings of SPIE. Volume 6511. (2007) 65110M 